\title{
Renormalization-scheme variation of a QCD perturbation expansion with tamed large-order behavior
}

\author{
Irinel Caprini \\ Horia Hulubei National Institute for Physics and Nuclear Engineering, \\ P.O.B. MG-6, 077125 Bucharest-Magurele, Romania
}

(Received 27 June 2018; published 19 September 2018)

\begin{abstract}
The renormalization-scheme and scale dependence of the truncated QCD perturbative expansions is one of the main sources of theoretical error of the standard model predictions, especially at intermediate energies. Recently, a class of renormalization schemes, parametrized by a single real number $C$, has been defined and investigated in the frame of the standard perturbation expansions in powers of the coupling. In the present paper we investigate the $C$-scheme variation of a Borel-improved QCD perturbation series, which implements information about the large-order divergent character of perturbation theory by means of an optimal conformal mapping of the Borel plane. In the new expansions, the powers of the strong coupling are replaced by a set of expansion functions with properties which resemble those of the expanded correlators, having in particular a singular behavior at the origin of the complex coupling plane. On the other hand, the new expansions have a tamed increase at high orders, as demonstrated by previous studies in the $\overline{\mathrm{MS}}$ renormalization scheme. Using as examples the Adler function and the hadronic decay width of the $\tau$ lepton, we investigate the properties of the Borel-improved expansions in the $C$-scheme, in comparison with the standard expansions in the $C$-scheme and the expansions in $\overline{\mathrm{MS}}$. The variation with the renormalization scale and the prescription for the choice of an optimal value of the parameter $C$ are discussed. The good large-order behavior of the Borel-improved expansions is proved also in the $C$-scheme, which is a further argument in favor of using them in applications of perturbative QCD at intermediate energies.
\end{abstract}

DOI: $10.1103 /$ PhysRevD.98.056016

\section{INTRODUCTION}

In the standard QCD perturbation theory, the finite-order approximations of physical quantities are renormalizationscale $(\mu)$ and renormalization-scheme (RS) dependent. For a truncated expansion of order $N$, the scale and scheme variations, being in principle $\mathcal{O}\left(\alpha_{s}^{N+1}(\mu)\right)$ corrections, are expected to be small at large scales due to asymptotic freedom. However, since the perturbative expansion in QCD is a divergent series, with coefficients growing factorially at large orders, the scale and scheme variation might be in practice quite large, especially at intermediate energies where the strong coupling $\alpha_{s}$ is not very small.

The quest for in some sense "optimal" scale and scheme is important for meaningful applications. There are several recipes [1-8] how to do that. The one proposed in [1] and known as the "principle of minimal sensitivity" (PMS) selects the scale and scheme by the condition of local scale

Published by the American Physical Society under the terms of the Creative Commons Attribution 4.0 International license. Further distribution of this work must maintain attribution to the author(s) and the published article's title, journal citation, and DOI. Funded by SCOAP ${ }^{3}$. and scheme invariance. Therefore, the PMS selects the point where the truncated approximant has locally the property which the all-order summation must have globally. A different, process-dependent recipe, known as "effective charge method" or "fastest apparent convergence" (FAC) criterion was proposed in [2], while the method advocated in [5-8], denoted as "principle of maximum conformality," chooses the scale such as to absorb in the coupling all the nonconformal dependence of the perturbative coefficients. Since the problem is difficult and has so far no generally accepted solution, perturbative computations are performed mainly in convenient schemes like the modified minimal subtraction $\overline{\mathrm{MS}}$ [9].

Recently, a new class of process-independent renormalization schemes depending on a single real parameter $C$ was defined in [10]. In Refs. [10-12], the properties of these schemes have been discussed using the perturbation expansion of the QCD Adler function and the $\tau$ hadronic width, and in [13] the class of $C$ schemes was investigated from the point of view of the maximum conformality principle [5-8].

In the present paper, we shall investigate the $C$-scheme in connection with the fact that the perturbation expansions in QCD are divergent series, the expansion coefficients of 
typical correlators growing factorially at large orders [14-17]. This is related to the fact that the QCD correlators as functions of $\alpha_{s}$ are singular at the origin of the complex $\alpha_{s}$ plane [14], which implies that the radius of convergence of the expansions in powers of the strong coupling $\alpha_{s}$ is zero.

Starting from the divergent character of the QCD perturbation series, a modified perturbation expansion was defined in [18] and was further investigated in [19-25] (for a recent review see [26]). In this approach, instead of the powers of the strong coupling, a new set of expansion functions is used, defined by means of an optimal (i.e., ensuring the best asymptotic rate of convergence) conformal mapping of the Borel complex plane. The properties of the new expansion functions resemble those of the expanded correlators, by exhibiting in particular a singular behavior at the origin of the $\alpha_{s}$ plane. On the other hand, the new expansions have a tamed increase at high orders. The good convergence properties of the new expansions have been demonstrated in [21,23,25] in the $\overline{\mathrm{MS}}$ renormalization scheme on mathematical models that simulate the physical Adler function.

The aim of the present work is to investigate the properties of these modified perturbation expansions in the class of renormalization schemes defined in [10]. As in [10-12], we use for illustration the Adler function and the hadronic width of the $\tau$ lepton. We start by recalling, in the next section, a few facts about the calculation of these quantities in perturbative QCD. In Sec. III we briefly review, following [10], the $C$-scheme definition of the QCD coupling. In Sec. IV we introduce the modified, nonpower perturbative expansions based on the conformal mapping of the Borel plane, and in Sec. V we rewrite them in the $C$-scheme. In Sec. VI we investigate the $C$-scheme variation of the modified perturbative expansions of the Adler function and the hadronic $\tau$ decay width. We also investigate the large-order behavior of the expansions, using for generating the large-order perturbative coefficients a model of the Adler function proposed in [27], which we present for completeness in the Appendix. The last section contains a summary and our conclusions.

\section{ADLER FUNCTION AND $\tau$ HADRONIC WIDTH IN PERTURBATIVE QCD}

We recall that the Adler function is the logarithmic derivative of the invariant amplitude of the two-current correlation tensor, $D(s)=-s d \Pi(s) / d s$, where $s$ is the momentum squared. As in Ref. [10] we shall consider the reduced function $\hat{D}(s)$ defined as:

$$
\hat{D}(s) \equiv 4 \pi^{2} D(s)-1 .
$$

From general principles of field theory, it is known that $\hat{D}(s)$ is an analytic function of real type [i.e., it satisfies the
Schwarz reflection property $\left.\hat{D}\left(s^{*}\right)=\hat{D}^{*}(s)\right]$ in the complex $s$ plane cut along the timelike axis for $s \geq 4 m_{\pi}^{2}$.

At large spacelike momenta $s<0$, the function $\hat{D}$ is given by the QCD perturbative expansion [27]

$$
\hat{D}\left(a_{\mu}\right)=\sum_{n \geq 1} a_{\mu}^{n} \sum_{k=1}^{n} k c_{n, k}\left(\ln \left(-s / \mu^{2}\right)\right)^{k-1},
$$

where $a_{\mu} \equiv \alpha_{s}\left(\mu^{2}\right) / \pi$ is the renormalized strong coupling in a certain RS at an arbitrary scale $\mu$. As in [10], we emphasize from now on the fact that $\hat{D}$ is a function of the QCD coupling $a_{\mu}$, the dependence on the momentum squared $s$ being implicit.

The leading coefficients $c_{n, 1}$ in (2) are obtained from the calculation of Feynman diagrams, while the remaining ones, $c_{n, k}$ with $k>1$ are obtained in terms of $c_{m, 1}$ with $m<n$ and the coefficients $\beta_{n}$ of the $\beta$ function, which governs the variation of the QCD coupling with the scale in each RS:

$$
-\mu \frac{d a_{\mu}}{d \mu} \equiv \beta\left(a_{\mu}\right)=\sum_{n \geq 1} \beta_{n} a_{\mu}^{n+1} .
$$

We recall that in mass-independent renormalization schemes the first two coefficients $\beta_{1}$ and $\beta_{2}$ are scheme invariant, depending only on the number $n_{f}$ of active flavors, while $\beta_{n}$ for $n \geq 3$ depend on the renormalization scheme. In $\overline{\mathrm{MS}}$, the known coefficients for $n_{f}=3$ are (cf. [28] and references therein):

$$
\begin{aligned}
& \beta_{1}=\frac{9}{2}, \quad \beta_{2}=8, \quad \beta_{3}=20.12, \\
& \beta_{4}=54.46, \quad \beta_{5}=268.16 .
\end{aligned}
$$

The Adler function was calculated in $\overline{\mathrm{MS}}$ to order $\alpha_{s}^{4}$, which makes it one of the most precisely known Green functions in QCD. For $n_{f}=3$ the leading coefficients $c_{n, 1}$ have the values (cf. [29] and references therein):

$$
\begin{aligned}
& c_{1,1}=1, \quad c_{2,1}=1.640, \\
& c_{3,1}=6.371, \quad c_{4,1}=49.076 .
\end{aligned}
$$

In the applications done in [10-12], an additional term was included, $c_{5,1}=283$, based on the estimate made in [27], and we shall adopt this value in the present work.

We shall consider also the perturbative expansion of the total $\tau$ hadronic width. The central observable is the ratio $R_{\tau}$ of the total hadronic branching fraction to the electron branching fraction, which can be expressed as

$$
R_{\tau}=3 S_{\mathrm{EW}}\left(\left|V_{u d}\right|^{2}+\left|V_{u s}\right|^{2}\right)\left(1+\delta^{(0)}+\ldots\right),
$$

where $S_{\mathrm{EW}}$ is an electroweak correction, $V_{u d}$ and $V_{u s}$ are Cabibbo-Kobayashi-Maskawa (CKM) matrix elements, 
and $\delta^{(0)}$ is the perturbative QCD contribution. As shown in [30-33], $\delta^{(0)}$ can be expressed, using analyticity, by an integral involving the values of the Adler function in the complex $s$ plane. In our normalization, this relation is [27]:

$$
\delta^{(0)}=\frac{1}{2 \pi i} \oint_{|s|=m_{\tau}^{2}} \frac{d s}{s}\left(1-\frac{s}{m_{\tau}^{2}}\right)^{3}\left(1+\frac{s}{m_{\tau}^{2}}\right) \hat{D}\left(a_{\mu}\right) .
$$

For the evaluation of $\delta^{(0)}$ one can either insert in the integral (7) the expansion (2) at a fixed scale and perform the integration of the coefficients with respect to $s$ along the circle, which gives in particular for $\mu=m_{\tau}$ :

$\delta_{\mathrm{FO}}^{(0)}=a_{\mu}+5.2 a_{\mu}^{2}+26.37 a_{\mu}^{3}+127.1 a_{\mu}^{4}+873.8 a_{\mu}^{5} \ldots$

Alternatively, as proposed in [33], one can take the variable scale $\mu^{2}=-s$ in (2) and insert in the integral (7) the renormalization-group improved expansion

$$
\hat{D}\left(a_{\mu}\right)=\sum_{n \geq 1} c_{n, 1} a_{\mu}^{n}, \quad \mu^{2}=-s
$$

with the running coupling calculated by the numerical integration of the renormalization-group equation (3) along the circle $|s|=m_{\tau}^{2}$, starting from the spacelike point $s=-m_{\tau}^{2}$. These alternatives ${ }^{1}$ are knows as "fixed-order perturbation theory" (FOPT) and "contour-improved perturbation theory" (CIPT). As remarked first in [27], contrary to the naive expectations, at the scale set by $m_{\tau}$ the difference between the predictions of these two summation procedures increased when an additional, five loop term, calculated in [29], was included in the expansion of the $\tau$ hadronic width.

The fixed-order series (2) is expected to have a poor convergence for $s$ near the timelike axis, where the $s$-dependent expansion coefficients become quite large. However, fortuitous cancellations of contributions to the integral (7) and the suppressing effect of the weight function in the integrand might favor the fixed-order series, leading to better results for $\delta^{(0)}$ calculated in FOPT than in CIPT. Many studies have been devoted to the difference between FO and CI summations, including the analysis of specific models for the Adler function and of the practical implications on the extraction of $\alpha_{s}\left(m_{\tau}^{2}\right)$ from data on hadronic $\tau$ decays (see [21-27,36-45] and the references therein).

\footnotetext{
${ }^{1}$ Another approach, proposed in [34], includes all the terms available from renormalization-group invariance and can be expressed as an effective expansion in powers of the one loop solution of Eq. (3). This summation was investigated in the case of the Adler function in $[24,35]$.
}

\section{THE $C$-SCHEME QCD COUPLING}

As discussed in [10], one can define a new coupling $\hat{a}_{\mu}$ by using the relation

$$
\begin{aligned}
\frac{1}{\hat{a}_{\mu}}+\frac{\beta_{2}}{\beta_{1}} \ln \hat{a}_{\mu}-\beta_{1} \frac{C}{2} & \equiv \beta_{1} \ln \frac{\mu}{\Lambda} \\
& =\frac{1}{a_{\mu}}+\frac{\beta_{2}}{\beta_{1}} \ln a_{\mu}-\beta_{1} \int_{0}^{a_{\mu}} \frac{\mathrm{d} a}{\bar{\beta}(a)}
\end{aligned}
$$

where $\Lambda$ is the scale-invariant QCD parameter and

$$
\frac{1}{\bar{\beta}(a)} \equiv \frac{1}{\beta(a)}-\frac{1}{\beta_{1} a^{2}}+\frac{\beta_{2}}{\beta_{1}^{2} a} .
$$

From (10) it is seen that $C$ incorporates the effects of all scheme-dependent terms $\beta_{n}$ with $n \geq 3$, contained in the function $\bar{\beta}(a)$. This relation implies also that the scale dependence of $\hat{a}_{\mu}$ is given by

$$
-\mu \frac{\mathrm{d} \hat{a}_{\mu}}{d \mu} \equiv \hat{\beta}\left(\hat{a}_{\mu}\right)=\frac{\beta_{1} \hat{a}_{\mu}^{2}}{\left(1-\frac{\beta_{2}}{\beta_{1}} \hat{a}_{\mu}\right)},
$$

and involves only the scheme-independent coefficients $\beta_{1}$ and $\beta_{2}$. Furthermore, as shown in $[11,12]$, the $C$ dependence of the coupling $\hat{a}_{\mu}$ is governed by the same schemeindependent function $\hat{\beta}$.

Given the coupling $a_{\mu}$ in a definite RS at a scale $\mu$, one can find from (10) the coupling $\hat{a}_{\mu}$ in the $C$-scheme at the same scale and a definite value of $C$. In order to solve numerically the equation, is convenient to write it as

$$
\frac{1}{\hat{a}_{\mu}}+\frac{\beta_{2}}{\beta_{1}} \ln \hat{a}_{\mu}=f\left(a_{\mu}, C\right),
$$

where $f\left(a_{\mu}, C\right)$ is a calculable function depending on the coupling $a_{\mu}$ and the constant $C$. The left-hand side of (13) is a convex function of $\hat{a}_{\mu}$, exhibiting a single minimum equal to 0.7549 at $\hat{a}_{\mu}=0.5625$, a steep increase towards small values of $\hat{a}_{\mu}$ and a slow logarithmic increase at large $\hat{a}_{\mu}$. Therefore, for values of $a_{\mu}$ and $C$ such that $f\left(a_{\mu}, C\right)>0.7549$, the equation (13) has a unique solution of interest, given by the intersection of the left branch of the function in the 1.h.s. with the horizontal line at coordinate $f\left(a_{\mu}, C\right)$.

As seen from Fig. 1 of [10], where the numerical solution is displayed for the input $\mu=m_{\tau}$ and $a_{\mu}=0.316(10) / \pi$ in the $\overline{\mathrm{MS}}$ scheme, the coupling $\hat{a}_{\mu}$ is a decreasing function of $C$. For further illustration we present in Fig. 1 the $C$-dependence of the coupling for the scale $\mu=0.61 m_{\tau}$, of interest for the analysis performed in Sec. VI. The coupling $a_{\mu}=0.442(20) / \pi$ in the $\overline{\mathrm{MS}}$ scheme was obtained by solving the RG equation with the input at $\mu=m_{\tau}$. In this 


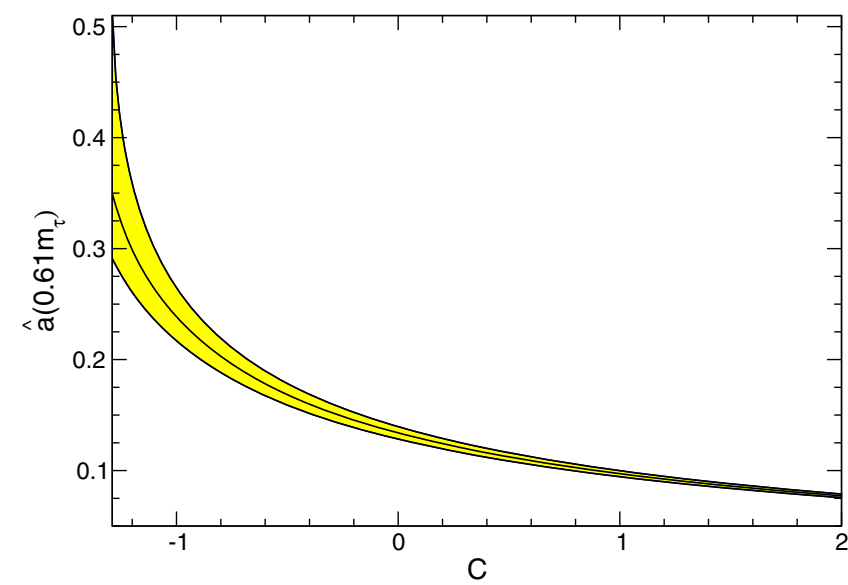

FIG. 1. The coupling $\hat{a}_{\mu}$ found by solving Eq. (13) for the scale $\mu=0.61 m_{\tau}$ and $a_{\mu}=0.442(20) / \pi$ in the $\overline{\mathrm{MS}}$ scheme, as a function of $\mathrm{C}$. The yellow band corresponds to the $\alpha_{s}$ uncertainty.

case, solutions of Eq. (13) exist only for $C>-1.293$, and one can see from Fig. 1 the large values of $\hat{a}_{\mu}$ near this limit of the validity region.

From (10) one can obtain also the perturbative relations between the coupling $a_{\mu}$ in a certain RS and the coupling $\hat{a}_{\mu}$ in the $C$-scheme:

$\hat{a}_{\mu}\left(a_{\mu}\right)=\sum_{n \geq 1} \xi_{n}(C) a_{\mu}^{n}, \quad a_{\mu}\left(\hat{a}_{\mu}\right)=\sum_{n \geq 1} \bar{\xi}_{n}(C) \hat{a}_{\mu}^{n}$

The explicit forms of these expansions for the $\overline{\mathrm{MS}}$ scheme are given in Eqs. (7) and (8) of [10]. From the comparison with the full solution, found numerically from (13), one can establish the range of $C$ where the perturbative expansions (14) are valid. For instance, as shown in [10], for $\mu=m_{\tau}$, when $a_{\mu}=0.316 / \pi$, the perturbative expansion breaks near $C=-2$. For higher values of the scales, when the coupling $a_{\mu}$ is smaller, the region of validity of (14) is extended to larger negative values of $C$, while for smaller scales the perturbative expansion breaks at negative values of $C$ closer to the origin.

The perturbative expansions in the $C$-scheme of the Adler function $\hat{D}$ and of the $\tau$ hadronic width have been investigated in [10-12]. For instance, the series (9) is rewritten as a series in powers of the $C$-scheme coupling $\hat{a}_{\mu}$ with $C$-dependent coefficients as

$$
\hat{D}\left(\hat{a}_{\mu}\right)=\sum_{n \geq 1} \hat{c}_{n, 1}(C) \hat{a}_{\mu}^{n}, \quad \mu^{2}=-s .
$$

Using in (9) the coefficients (5) and the estimate $c_{5,1}=283$, this expansion reads [10]

$$
\begin{aligned}
\hat{D}\left(\hat{a}_{\mu}\right)= & \hat{a}_{\mu}+(1.64+2.25 C) \hat{a}_{\mu}^{2} \\
& +\left(7.68+11.38 C+5.06 C^{2}\right) \hat{a}_{\mu}^{3} \\
& +\left(61.06+72.08 C+47.40 C^{2}+11.4 C^{3}\right) \hat{a}_{\mu}^{4} \\
& +\left(348.5+677.7 C+408.6 C^{2}+162.5 C^{3}\right. \\
& \left.+25.6 C^{4}\right) \hat{a}_{\mu}^{5}+\ldots
\end{aligned}
$$

In the same way, from (8) it follows that the expansion of $\delta_{\mathrm{FO}}^{(0)}$ in powers of the $C$-scheme coupling $\hat{a}_{\mu}$ at the scale $\mu=m_{\tau}$ is [10]

$$
\begin{aligned}
\delta_{\mathrm{FO}}^{(0)}\left(\hat{a}_{\mu}\right)= & \hat{a}_{\mu}+(5.20+2.25 C) \hat{a}_{\mu}^{2} \\
& +\left(27.7+27.4 C+5.1 C^{2}\right) \hat{a}_{\mu}^{3} \\
& +\left(148.4+235.5 C+101.5 C^{2}+11.4 C^{3}\right) \hat{a}_{\mu}^{4} \\
& +\left(789.6+1754.4 C+1240.4 C^{2}+324.8 C^{3}\right. \\
& \left.+25.6 C^{4}\right) \hat{a}_{\mu}^{5}+\ldots
\end{aligned}
$$

The studies performed in [10-12] showed that the predictions of the perturbation expansions in the $C$-scheme are comparable to those in the $\overline{\mathrm{MS}}$ scheme. In particular, the difference between the FOPT and CIPT predictions for $\delta^{(0)}$ is not resolved by the $C$-scheme, and the higher-order divergence of the expansions manifests itself to the same or even to a larger extent.

The works quoted above investigated the scheme dependence of the standard perturbation expansions of the QCD correlators in powers of the strong coupling. In this work we shall investigate the properties of the $C$-scheme using a modified QCD perturbative expansion, defined in $[18,21,23]$, which we briefly review in the next section.

\section{PERTURBATION EXPANSIONS WITH TAMED HIGH-ORDER BEHAVIOR}

The starting point is the remark that the perturbation expansion (9) of the Adler function has a zero radius of convergence, the coefficients $c_{n, 1}$ increasing like $n$ ! at large $n$. It is convenient to define the Borel transform by

$$
B(u)=\sum_{n=0}^{\infty} b_{n} u^{n}, \quad b_{n}=\frac{c_{n+1,1}}{\beta_{0}^{n} n !}
$$

where we used the standard notation $\beta_{0}=\beta_{1} / 2$. From (18) one can derive the formal Laplace-Borel integral representation

$$
\hat{D}\left(a_{\mu}\right)=\frac{1}{\beta_{0}} \int_{0}^{\infty} \exp \left(\frac{-u}{\beta_{0} a_{\mu}}\right) B(u) d u .
$$

As it is known [15,17], the large-order increase of the coefficients of the perturbation series is encoded in the 
singularities of the Borel transform $B(u)$ in the complex $u$ plane. In the particular case of the Adler function, $B(u)$ has singularities on the semiaxis $u \geq 2$, denoted as infrared (IR) renormalons, and for $u \leq-1$, denoted as ultraviolet (UV) renormalons. The names indicate the regions in the Feynman integrals responsible for the appearance of the corresponding singularities. Other singularities, at larger values on the positive real axis, are due to specific field configurations known as instantons. Apart from the two cuts along the lines $u \geq 2$ and $u \leq-1$, it is assumed that no other singularities are present in the complex $u$ plane [15].

Due to the singularities of $B(u)$ for $u \geq 2$, the LaplaceBorel integral (19) is not defined and requires a regularization. Several prescriptions have been adopted for various QCD correlators, in particular the principal value $(\mathrm{PV})$ prescription defined as $[16,17]$

$$
\begin{aligned}
& \frac{1}{\beta_{0}} \mathrm{PV} \int_{0}^{\infty} e^{-\frac{u}{\beta_{0} a_{\mu}}} B(u) d u \\
& \quad \equiv \frac{1}{2 \beta_{0}}\left[\int_{C_{+}} e^{-\frac{u}{\beta_{0} a_{\mu}}} B(u) d u+\int_{C_{-}} e^{-\frac{u}{\beta_{0} a_{\mu}}} B(u) d u\right],
\end{aligned}
$$

where $C_{ \pm}$are lines parallel to the positive real axis, slightly displaced above (below) it. As discussed in [46], this prescription is suitable from the point of view of the momentum-plane analyticity properties that must be satisfied by QCD correlators like the Adler function. In particular, it preserves Schwarz reflection principle $\hat{D}\left(s^{*}\right)=\hat{D}^{*}(s)$ in the complex $s$ plane and leads to real values for the Adler function on the spacelike axis $s<0$.

Of course, even after choosing a proper regularization, an uncertainty $\delta_{\text {ren }}$ related to the renormalon treatment still remains. A natural choice for this uncertainty is the difference between two regularization prescriptions, which can be taken as

$$
\begin{aligned}
& \frac{1}{\beta_{0}} \delta_{\text {ren }}\left\{\int_{0}^{\infty} e^{-\frac{u}{\beta_{0} a_{\mu}}} B(u) d u\right\} \\
& \quad \equiv \frac{1}{2 i \beta_{0}}\left[\int_{C_{+}} e^{-\frac{u}{\beta_{0} a_{\mu}}} B(u) d u-\int_{C_{-}} e^{-\frac{u}{\beta_{0} a_{\mu}}} B(u) d u\right] .
\end{aligned}
$$

This prescription has been used for assessing the uncertainty of the model for the Adler function proposed in [27] and reviewed in the Appendix, and we shall use it for some of the expansions investigated in this work.

The singularities of $B(u)$ set a limitation on the convergence region of the power expansion (18): this series converges only inside the circle $|u|=1$ which passes through the nearest singularity, namely the first UV renormalon. As it is known, the domain of convergence of a power series can be increased by expanding in powers of another variable, which performs the conformal mapping of the complex plane of the original variable (or a part of it) onto a disk.

The conformal mapping method was introduced in particle physics in [47-49] for improving the convergence of the power series used for the representation of scattering amplitudes. The new series converges in a larger region, well beyond the disk of convergence of the original expansion, and also has an increased asymptotic convergence rate at points lying inside this disk. An important result proved in $[47,49]$ is that the asymptotic convergence rate is maximal if the new variable maps the entire holomorphy domain of the expanded function onto the unit disk (for a detailed proof see [23]). This particular variable is known in the literature as the "optimal conformal mapping."

For QCD, the method of conformal mapping is not applicable to the formal perturbative series of $\hat{D}$ in powers of $a_{\mu}$, because $\hat{D}$ is singular at the origin of the coupling plane. ${ }^{2}$ However, the method can be applied in a straightforward way to the Borel transform $B(u)$, which is holomorphic in a region containing the origin $u=0$ of the Borel complex plane and can be expanded in powers of the Borel variable as in (18).

The use of a conformal mapping of the Borel plane was suggested in [16] in order to reduce or eliminate the ambiguities (power corrections) due to the large momenta in the Feynman integrals. This is achieved by a variable that maps on a unit disk the $u$ complex plane cut only along the line $u \leq-1$. As shown however in [18], the conformal mapping proposed in [16] (and used further in [52,53]) is not optimal in the sense defined above. The optimal mapping, which ensures the convergence of the corresponding power series in the entire doubly-cut Borel plane, is given by the function [18]

$$
\tilde{w}(u)=\frac{\sqrt{1+u}-\sqrt{1-u / 2}}{\sqrt{1+u}+\sqrt{1-u / 2}} .
$$

One can check that the function $\tilde{w}(u)$ maps the complex $u$ plane cut along the real axis for $u \geq 2$ and $u \leq-1$ onto the interior of the circle $|w|=1$ in the complex plane $w \equiv \tilde{w}(u)$, such that the origin $u=0$ of the $u$ plane corresponds to the origin $w=0$ of the $w$ plane, and the upper (lower) edges of the cuts are mapped onto the upper (lower) semicircles in the $w$ plane. By the mapping (22), all the singularities of the Borel transform, the UV and IR

\footnotetext{
${ }^{2}$ In the so-called "order-dependent" conformal mappings, which have been used for expansions in powers of the coupling [50,51], the singularity is shifted away from the origin by a certain amount at each finite perturbative order, and tends to the origin only when an infinite number of terms are considered.
} 
renormalons, are pushed on the boundary of the unit disk in the $w$ plane, all at equal distance from the origin. Therefore, the expansion of $B(u)$ in powers of the variable $w \equiv \tilde{w}(u)$ :

$$
B(u)=\sum_{n \geq 0} c_{n} w^{n}, \quad w=\tilde{w}(u)
$$

converges in a larger domain that the original series (18).

The expansion can be further improved by exploiting also the fact that the nature of the leading singularities of $B(u)$ in the Borel plane is known: near the first branch points, $u=-1$ and $u=2, B(u)$ behaves like

$$
B(u) \sim \frac{r_{1}}{(1+u)^{\gamma_{1}}} \quad \text { and } \quad B(u) \sim \frac{r_{2}}{(1-u / 2)^{\gamma_{2}}},
$$

respectively, where the residues $r_{1}$ and $r_{2}$ are not known, but the exponents $\gamma_{1}$ and $\gamma_{2}$ have been calculated using renormalization-group invariance [16,27,54]. For $n_{f}=3$, their values are

$$
\gamma_{1}=1.21, \quad \gamma_{2}=2.58
$$

The knowledge of the nature of the first IR renormalon of the Adler function was exploited for the first time in [53], where the Borel transform was multiplied by the factor $(1-u / 2)^{\gamma_{2}}$ and the product was expanded as a power series. ${ }^{3}$ But the multiplication with other factors is possible. As discussed in [21,23], while the optimal conformal mapping (22) is unique, there is no unique prescription to implement the knowledge provided by (24). For instance, using the fact that

$$
(1+w)^{2 \gamma_{1}} \sim(1+u)^{\gamma_{1}}, \quad(1-w)^{2 \gamma_{2}} \sim(1-u / 2)^{\gamma_{2}},
$$

for $u$ near -1 and 2, respectively, one can construct these factors in terms of the variable $w$ defined in (22).

It is easy to see that, although the product of $B(u)$ with these suitable factors is finite at $u=-1$ and $u=2$, it still has singularities (branch-points) at these points, generated by the terms of $B(u)$ which are holomorphic there. So, the procedure does not eliminate the singularities, but only makes them milder. This is why the procedure was denoted in [53] as "singularity softening" and the factors used to multiply the Borel function are referred to as "softening factors."

Since the product of $B(u)$ with the softening factors has still branch-points at $u=-1$ and $u=2$, the optimal conformal mapping for the expansion of the product is the function $w$ defined in (22). Using this remark, we shall adopt the expansion

\footnotetext{
${ }^{3}$ A similar procedure to account for the first IR renormalon was used in $[55,56]$ for other QCD correlators.
}

$$
B(u)=\frac{1}{(1+w)^{2 \gamma_{1}}(1-w)^{2 \gamma_{2}}} \sum_{n \geq 0} d_{n} w^{n},
$$

proposed in [21] and shown in [23] on mathematical models to have good convergence properties at high orders. In order to estimate the effect of each softening factor in (27), it is instructive to consider also the alternative expansions

$$
\begin{aligned}
& B(u)=\frac{1}{(1-w)^{2 \gamma_{2}}} \sum_{n \geq 0} d_{n}^{\prime} w^{n}, \\
& B(u)=\frac{1}{(1+w)^{2 \gamma_{1}}} \sum_{n \geq 0} d_{n}^{\prime \prime} w^{n},
\end{aligned}
$$

in which only the first IR/UV renormalon has been softened, respectively.

Actually, since the softening factors remove the divergencies at $u=-1$ and $u=2$, leaving only mild singularities at these points, one can expand the product in powers of other conformal variables, which take into account only the position of the more distant singularities of $B(u)$. Such mappings have been considered in [23,57]. Moreover, in [23] a detailed study of various softening factors and conformal mappings has been performed.

As an extreme case, an expansion in powers of the original variable $u$ can be used after "softening" the first singularities. To assess in more detail the importance of various factors, we shall consider the expansions

$$
\begin{gathered}
B(u)=\frac{1}{(1-u / 2)^{\gamma_{2}}} \sum_{n \geq 0} b_{n}^{\prime} u^{n}, \\
B(u)=\frac{1}{(1+u)^{\gamma_{1}}} \sum_{n \geq 0} b_{n}^{\prime \prime} u^{n},
\end{gathered}
$$

and

$$
B(u)=\frac{1}{(1-u / 2)^{\gamma_{2}}(1+u)^{\gamma_{1}}} \sum_{n \geq 0} b_{n}^{\prime \prime \prime} u^{n},
$$

where only the first IR/UV renormalons and both are taken into account, respectively. One can expect that at low orders the effect of including the known behavior near the first renormalons is important, while at high orders the singularities which remain in the product, even if they are mild, will deteriorate the convergence unless a conformal mapping is used.

By inserting the expansions (23), (27), (28), (29), (30) and (31) in the Borel-Laplace integral (19), we can define new perturbative series for the Adler function. When the expansion of $B(u)$ contains a singularity along the integration range, as in (23), (27), (28) and (30), we shall adopt 
the PV prescription defined in (20). Thus, for instance, the expansion (27) leads to

$$
\hat{D}\left(a_{\mu}\right)=\sum_{n \geq 0} d_{n} \mathcal{W}_{n}\left(a_{\mu}\right)
$$

where the expansion functions are

$$
\mathcal{W}_{n}\left(a_{\mu}\right)=\frac{1}{\beta_{0}} \mathrm{PV} \int_{0}^{\infty} \frac{\mathrm{e}^{-\frac{u}{\beta_{0} a_{\mu}}}(\tilde{w}(u))^{n}}{(1+\tilde{w}(u))^{2 \gamma_{1}}(1-\tilde{w}(u))^{2 \gamma_{2}}} d u
$$

We emphasize that the definition (33) implies the permutation of the summation and the integration, which is not a trivial step, therefore (33) represents a genuinely new perturbation expansion in QCD.

The properties of the new expansions like (33) have been investigated in detail in $[19,20,23]$, and we briefly summarize them here. By construction, when reexpanded in powers of $a_{\mu}$, the series (33) reproduces the expansion (9) with the coefficients $c_{n, 1}$ known from Feynman diagrams. The expansion functions $\mathcal{W}_{n}\left(a_{\mu}\right)$ are singular at $a_{\mu}=0$ and have divergent expansions when expanded in powers of $a_{\mu}$, resembling the expanded function $\hat{D}\left(a_{\mu}\right)$ itself. On the other hand, the expansion (33) has a tamed behavior at high orders, and, under certain conditions, it may even converge in a domain of the $s$-plane.

Since the expansion functions $\mathcal{W}_{n}\left(a_{\mu}\right)$ defined in (34) are no longer powers of the coupling $a_{\mu}$, the new expansion (33) can be viewed as a "nonpower perturbation theory" (NPPT) [23]. We shall also refer to it as "Borel-improved" expansion, to emphasize the fact that (33) is defined by the analytic continuation of the Borel series (18) outside the original convergence disk $|u|<1$, to the whole Borel plane up to its cuts.

We presented above the steps leading to the new expansion (33) starting from the renormalization-group improved expansion (9), but similar steps can be followed starting from the fixed-order expansion (2), when the scale $\mu^{2}$ is different from the energy squared $-s$. The explicit formulas are given in [23]. By inserting in (19) the expansions (23), (28), (29), (30) and (31) of the Borel transform, we obtain also the alternative expansions of $\hat{D}$ which will be investigated in our study.

\section{BOREL-IMPROVED EXPANSIONS IN THE $C$-SCHEME}

The nonpower expansions defined above have been investigated up to now in the $\overline{\mathrm{MS}}$ renormalization scheme. However, the construction presented in the previous section is general and can be performed in any renormalization scheme. Starting from the expansion (15) of the Adler function in powers of the $C$-scheme coupling $\hat{a}_{\mu}$, we define the corresponding Borel transform as

$$
\hat{B}(u, C)=\sum_{n=0}^{\infty} \hat{b}_{n}(C) u^{n}, \quad \hat{b}_{n}=\frac{\hat{c}_{n+1,1}(C)}{\beta_{0}^{n} n !},
$$

and obtain the formal Laplace-Borel integral representation

$$
\hat{D}\left(\hat{a}_{\mu}\right)=\frac{1}{\beta_{0}} \int_{0}^{\infty} \exp \left(\frac{-u}{\beta_{0} \hat{a}_{\mu}}\right) \hat{B}(u, C) d u .
$$

A useful remark is that the position and the nature of the first singularities of the Borel transform in the $u$ plane depend only on the first two coefficients, $\beta_{1}$ and $\beta_{2}$, of the $\beta$ function, which are scheme-independent [16,27,54]. It follows that, for every $C$, the first singularities of the function $\hat{B}(u, C)$ are situated at $u=-1$ and $u=2$, and the nature of the singularities is given by (24). Therefore, we can use for $\hat{B}(u, C)$ the expansions similar to those written in (23) and (27)-(32) in the $\overline{\mathrm{MS}}$ scheme. In particular, we write the expansion

$$
\hat{B}(u, C)=\frac{1}{(1+w)^{2 \gamma_{1}}(1-w)^{2 \gamma_{2}}} \sum_{n \geq 0} \hat{d}_{n}(C) w^{n},
$$

similar to (27), the only difference being that now the coefficients $\hat{d}_{n}$ depend on $C$. By inserting this expansion into (36), we define the Borel-improved expansion of the Adler function in the $C$-scheme by

$$
\hat{D}\left(\hat{a}_{\mu}\right)=\sum_{n \geq 0} \hat{d}_{n}(C) \hat{\mathcal{W}}_{n}\left(\hat{a}_{\mu}\right)
$$

where the expansions functions $\hat{\mathcal{W}}_{n}\left(\hat{a}_{\mu}\right)$ are obtained from (34) by formally replacing $a_{\mu}$ with the $C$-dependent coupling $\hat{a}_{\mu}$.

For illustration, we list the first coefficients $\hat{d}_{n}(C)$ appearing in (38):

$\hat{d}_{0}=1$,

$\hat{d}_{1}=-0.80+2.67 C$,

$\hat{d}_{2}=1.33+2.46 C+3.56 C^{2}$,

$\hat{d}_{3}=10.69+2.31 C+8.15 C^{2}+3.16 C^{3}$,

$\hat{d}_{4}=1.15+23.44 C+8.37 C^{2}+11.02 C^{3}+2.11 C^{4}$.

In a similar way, starting from the expansion (17) of $\delta_{\mathrm{FO}}^{(0)}$ in the $C$-scheme, we can define the improved series in terms of the same set of functions $\hat{\mathcal{W}}_{n}\left(\hat{a}_{\mu}\right)$, since from the definition (8) it follows that the position and the nature 
of the first singularities of the Borel transform of $\delta_{\mathrm{FO}}^{(0)}$ are the same as those of the Adler function, So, we can write

$$
\delta_{\mathrm{FO}}^{(0)}\left(\hat{a}_{\mu}\right)=\sum_{n \geq 0} \hat{\delta}_{n}(C) \hat{\mathcal{W}}_{n}\left(\hat{a}_{\mu}\right)
$$

where $\mu=m_{\tau}$ and the first coefficients $\hat{\delta}_{n}(C)$ are

$$
\begin{aligned}
& \hat{\delta}_{0}=1, \\
& \hat{\delta}_{1}=3.42+2.67 C \\
& \hat{\delta}_{2}=6.62+13.72 C+3.56 C^{2}, \\
& \hat{\delta}_{3}=4.96+31.82 C+23.16 C^{2}+3.16 C^{3}, \\
& \hat{\delta}_{4}=-14.22+29.32 C+65.63 C^{2}+24.36 C^{3}+2.11 C^{4} .
\end{aligned}
$$

In CIPT, the calculation involves the numerical integration (7) of the expansion (38), written as:

$$
\begin{aligned}
\delta_{\mathrm{CI}}^{(0)}\left(\hat{a}_{m_{\tau}}\right)= & \sum_{n \geq 0} \hat{d}_{n}(C) \\
& \times \frac{1}{2 \pi i} \oint_{|s|=m_{\tau}^{2}} \frac{d s}{s}\left(1-\frac{s}{m_{\tau}^{2}}\right)^{3}\left(1+\frac{s}{m_{\tau}^{2}}\right) \hat{\mathcal{W}}_{n}\left(\hat{a}_{\mu}\right),
\end{aligned}
$$

where the coefficients $\hat{d}_{n}$ are given in (39) and the expansion functions $\hat{\mathcal{W}}_{n}$, defined below (38), depend on the running coupling $\hat{a}_{\mu}$ at the scale $\mu^{2}=m_{\tau}^{2} \exp (i(\phi-\pi))$, $\phi \in(0,2 \pi)$, which is calculated along the circle by integrating the renormalization-group equation (12) in the $C$-scheme, starting from a given value at the scale $\mu=m_{\tau}$. Therefore, the whole expansion depends only on the $C$-scheme coupling $\hat{a}_{\mu}$ at the scale $\mu=m_{\tau}$.

\section{RESULTS}

\section{A. Adler function}

As a first application, we calculate the Adler function (1) at the spacelike point $s=-m_{\tau}^{2}$ using the Borel-improved expansion in the $C$-scheme. Following Ref. [10], we take first the scale $\mu=m_{\tau}$, when the perturbative expansion (2) writes as the renormalization-group improved series (9). Other scales will be also considered below. We use the value $\alpha_{s}\left(m_{\tau}^{2}\right)=0.316 \pm 0.010$ in the $\overline{\mathrm{MS}}$ scheme, which follows from the PDG value of the strong coupling at the scale $\mu=m_{Z}$ [58]. The corresponding $C$-dependent value of the coupling $\hat{a}_{m_{\tau}}$ was obtained by numerically solving Eq. (13), as explained in Sec. III.

In Fig. 2, we show the variation with $C$ of the Borelimproved expansions in the $C$-scheme, given in Eqs. (38) and (39), for $C$ in the range $(-2,2)$ : the central black line

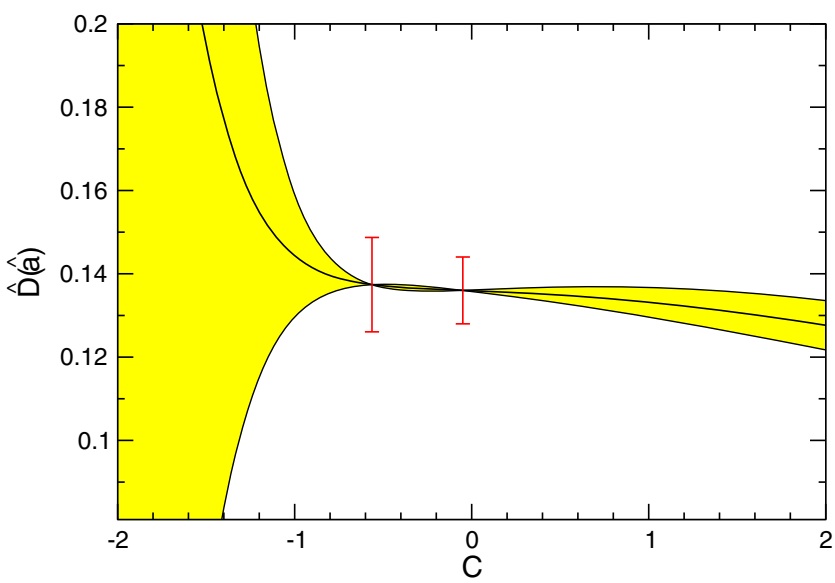

FIG. 2. Borel-improved expansions in the $C$-scheme of the Adler function $\hat{D}$ at $s=-m_{\tau}^{2}$. The central line is the expansion to order $\alpha_{s}^{5}$. The yellow band is obtained by either removing or doubling the last term. Marked in red are the points where the last term of the expansion vanishes and the magnitude of the last nonvanishing term is shown.

represents the expansion to order $\alpha_{s}^{5}$, and the lines delimiting the yellow region are obtained by either removing or doubling the coefficient $\hat{d}_{4}$ given in (39).

The three curves exhibit a plateau where the expansions are stable with respect to the variation of $C$. There are two points, represented in red, where the three expansions coincide: the rightmost point, $C_{0}=-0.05$, is the only real solution of the equation $\hat{d}_{4}=0$ in the range $(-2,2)$. At the other point, $C_{0}^{\prime}=-0.56$, the expansion function $\hat{\mathcal{W}}_{4}$ itself vanishes, a phenomenon which can take place since the expansion functions are no longer simple powers of the coupling $\hat{a}_{\mu}$. At these points we indicate the magnitude of the previous nonvanishing term, $\hat{d}_{3} \hat{\mathcal{W}}_{3}$.

In Ref. [10], the point where the three expansions coincide has been selected as the optimal value of the parameter $C$. If there are more values of $C$ which satisfy this condition, the optimal one was taken such as to lead to a minimal value for the last nonvanishing term, which was interpreted as a truncation error. ${ }^{4}$ In the present work, we shall consider for the beginning the same prescription, which is in the spirit of the "fastest apparent convergence" [2] and "minimum sensitivity" [1] principles. In our case this point is $C_{0}=-0.05$, which leads to the central value $\hat{D}=0.1364$. For comparison, we note that the Borelimproved expansion to $O\left(\alpha_{s}^{5}\right)$ in the $\overline{\mathrm{MS}}$ scheme gives the close value 0.1360 , while the standard expansions in the $C$-scheme and in $\overline{\mathrm{MS}}$ lead to the values 0.1343 and 0.1316 , respectively [10].

\footnotetext{
${ }^{4} \mathrm{~A}$ bit surprising is the fact (discussed in the last section), that the optimal $C$ and the error prescription proposed in Ref. [10] only work at 5 loops. In fact, the 5 th order has not been computed analytically, and a numerical estimate is used.
} 
The main sources of theoretical errors to be attached to these values are the uncertainty of the strong coupling $\alpha_{s}\left(m_{\tau}^{2}\right)$, the effect of truncating the perturbative series at a finite order and the variation of the renormalization scale. The first error can be easily calculated in each case. The truncation error was taken in [10] as the magnitude of the last nonvanishing term kept in the expansion. For the Borelimproved expansions the last nonvanishing terms turn out to be equal to 0.0080 and 0.0030 in the $C$ and $\overline{\mathrm{MS}}$ schemes, respectively, while for the standard expansions the corresponding values are 0.0070 and 0.0029 . Actually, no definite way for assessing the effect of the unknown higher-order terms in the perturbative expansion exists. The choice made in [10], in the spirit of asymptotic expansions, may be affected by numerical accidental cancellations leading to values too small for the last coefficient.

Following [10], we adopted in the above calculation the scale $\mu=m_{\tau}$, when all the logarithms in the expansion (2) are summed leading to the renormalization-group improved series (9). However, it is instructive to investigate also other scales. Following [36], we parametrized the scale dependence by writing $\mu^{2}=-\xi s$ with $\xi=1 \pm 0.63$. A similar range of $\mu$ has been adopted in [42]. In particular, for the point $s=-m_{\tau}^{2}$ used in the calculation of the Adler function, the scale is written as $\mu=\sqrt{\xi} m_{\tau}$, and varies between $0.61 m_{\tau}$ and $1.28 m_{\tau}$.

By comparing the perturbative expansion (14) with the full solution determined numerically from (13), one can see that for scales $\mu$ larger than $m_{\tau}$, when the coupling $\alpha_{s}$ is smaller, the perturbative expansion is valid on a larger interval, extending to the left of $C=-2$. On the other hand, as seen from Fig. 1, for smaller $\mu$ the coupling is larger and the validity of the perturbative expansion (14) breaks down at values of $C$ closer to 0 .

In Fig. 3 we show the Borel-improved expansions in the $C$-scheme of the Adler function $\hat{D}$ at $s=-m_{\tau}^{2}$, for the scale $\mu=m_{\tau}$ and for the extreme values $\mu=0.61 m_{\tau}$ and $\mu=1.28 m_{\tau}$ of the range considered. We show also in each case the points where the last term of the expansion vanishes and the magnitude of the last nonvanishing term. For $\mu=m_{\tau}$ the curve and the optimal point coincide with those given in Fig. 2.

In order to quantify the variation with the scale, we can compare the results for different scales at a fixed value of $C$, namely the optimal $C$ determined for $\mu=m_{\tau}$ (the point marked in red). A different prescription would be to compare the optimal values for each scale, obtained with the corresponding optimal values of $C$. As seen from Fig. 3, for the Adler function the first definition appears to give reasonable estimates of the errors, while with the second prescription the errors appear to be underestimated. Therefore, for the Borel-improved expansion in the $C$-scheme we adopt the first prescription for the error.

On the other hand, for the standard expansions in the $C$-scheme, where the optimal value of $C$ for $\mu=m_{\tau}$ is

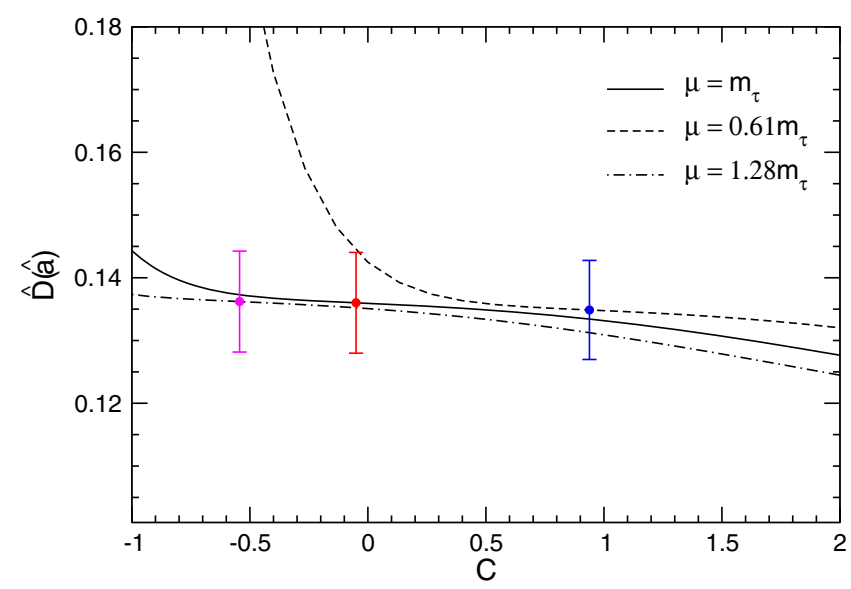

FIG. 3. Borel-improved expansions to order $\alpha_{s}^{5}$ in the $C$-scheme of the Adler function $\hat{D}$ at $s=-m_{\tau}^{2}$, for the scales $\mu=m_{\tau}, \mu=$ $0.61 m_{\tau}$ and $\mu=1.28 m_{\tau}$, as functions of $C$. We show in each case the optimal points where the last term of the expansion vanishes and the magnitude of the last nonvanishing term.

$C_{0}=-0.78$ [10], the perturbative expansion for $\mu$ towards the lower end of the range is not well behaved. In this case, one can either use for low values of $\mu$ the second prescription mentioned above, or reduce the interval of scale variation. This ambiguity may induce some distortions in the error estimate, which must be taken with caution in these cases. ${ }^{5}$

By including all sources of error, we obtain the result of the Borel-improved expansion in $C$-scheme as

$$
\hat{D}=0.1364 \pm 0.0080_{-0.0012}^{+0.0078} \pm 0.0061,
$$

where the first error is the magnitude of the last nonvanishing term in the expansion, the second is the uncertainty due to scale variation and the third is due to the uncertainty in $\alpha_{s}\left(m_{\tau}^{2}\right)$.

For comparison we note that to the same order the Borelimproved expansion in the $\overline{\mathrm{MS}}$ scheme gives

$$
\hat{D}=0.1360 \pm 0.0030_{-0.0010}^{+0.0034} \pm 0.0061,
$$

where the significance of the uncertainties is the same. The standard expansion in the $C$-scheme gives

$$
\hat{D}=0.1343 \pm 0.0070_{-0.0016}^{+0.0001} \pm 0.0067,
$$

where the error due to the scale variation has been estimated from the differences of the optimal values at different scales, and might be underestimated. For the standard expansion in $\overline{\mathrm{MS}}$ the result is

\footnotetext{
${ }^{5}$ The problems encountered when varying the scale indicate that a more elaborate prescription for the optimal $C$ than that proposed in [10] may be necessary. We will make some comments on this in the final section.
} 


$$
\hat{D}=0.1316 \pm 0.0029_{-0.0030}^{+0.0029} \pm 0.0060 \text {. }
$$

We note finally that, due to the conformal mapping (22) and the softening factors (when present), the definition of the Borel-improved expansions requires a regularization prescription of the Borel-Laplace integral. For calculating the central values we adopted the PV prescription (20), which has the advantage of preserving Schwarz reflection principle and leads to real values for the Adler function on the spacelike axis. Since the PV prescription is part of the definition of the Borel-improved expansions, we do not include the regularization ambiguity as an additional error. For completeness, we mention that the definition (21) leads to similar values, equal to 0.0098 and 0.0089 , respectively, for the prescription ambiguity of the Borel-improved expansions in the $C$ and $\overline{\mathrm{MS}}$ schemes.

\section{B. $\tau$ hadronic width}

We consider now the calculation of the QCD correction $\delta^{(0)}$ to the $\tau$ hadronic width (6). As discussed in Sec. II, there are two standard summation methods, CIPT and FOPT, which differ essentially by the choice of the renormalization scale in performing the integral (7) along the circle in the $s$ plane. The difference between FOPT and CIPT in the $\overline{\mathrm{MS}}$ scheme is the main source of uncertainty in the extraction of the strong coupling from hadronic $\tau$ decays. As discussed in Ref. [10], this difference persists also in the $C$ scheme. In the present subsection we investigate the problem using the Borel-improved FO and CI expansions of $\delta^{(0)}$ in the $C$ scheme, discussed in Sec. V.

The Borel-improved FO expansion of $\delta^{(0)}$ in the $C$-scheme is given in Eqs. (40) and (41). According to standard practice in the perturbative calculations of $\tau$ hadronic width, the scale was first fixed at $\mu=m_{\tau}$. As in the previous subsection, one explored also other scales, taking $\mu^{2}=-\xi$ s with $\xi=1 \pm 0.63$ [36].

In Fig. 4 we show the Borel-improved FO expansion of $\delta^{(0)}$ in the $C$ scheme to order $\alpha_{s}^{5}$ as central line, and the curves obtained by either doubling or removing the last term in the expansion, which delimit the yellow band. As seen from the figure, there are three points where the curves coincide: the leftmost and the rightmost ones are the solutions of the equation $\hat{\delta}_{4}=0$, while the middle point is the solution of the equation $\hat{\mathcal{W}}_{4}=0$, encountered already in Fig. 2. It turns out that the magnitude of the last nonvanishing term, $\hat{\delta}_{3} \hat{\mathcal{W}}_{3}$, is minimal for the leftmost point, $C_{0}=-1$, which we adopt as optimal. This leads to the prediction

$$
\delta_{\mathrm{FO}}^{(0)}=0.2207 \pm 0.0039_{-0.0082}^{+0.0003} \pm 0.0195,
$$

where the first error is the magnitude of the last nonvanishing term in the expansion, the second one is obtained from the scale variation and the third accounts for the

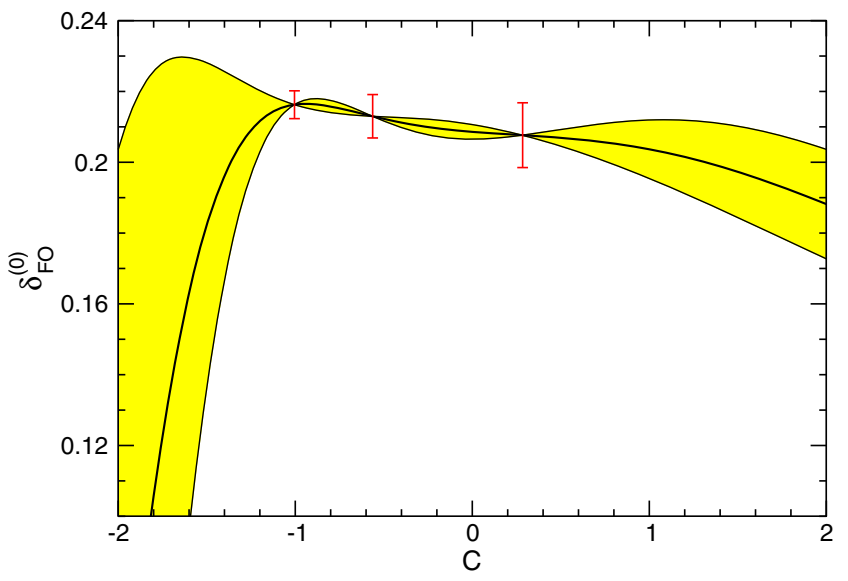

FIG. 4. Borel-improved expansion of $\delta_{\mathrm{FO}}^{(0)}$ to order $O\left(\alpha_{s}^{5}\right)$ in the $C$ scheme as a function of $C$. The yellow band arises from either removing or doubling the last term in the expansion. In red are marked the points where the last term in the expansion vanishes, and the magnitude of the previous one is indicated.

uncertainty in the coupling. We note that we encountered the situation mentioned above for the Adler function in the standard $C$-scheme, i.e., the optimal $C_{0}$ for $\mu=m_{\tau}$ is close to -1 , where the expansions at low scales $\mu$ are not wellbehaved. Therefore, the errors due to scale variation quoted in (47), calculated by taking the differences of the optimal values for each scale, might be underestimated.

For comparison, the Borel-improved expansion in the $\overline{\mathrm{MS}}$ scheme to $O\left(\alpha_{s}^{5}\right)$ gives

$$
\delta_{\mathrm{FO}}^{(0)}=0.2104 \pm 0.0031_{-0.0020}^{+0.0108} \pm 0.0136,
$$

where the significance of the terms is the same, while the standard expansion in the $C$-scheme predicts

$$
\delta_{\mathrm{FO}}^{(0)}=0.2047 \pm 0.0034_{-0.0059}^{+0.0002} \pm 0.0133,
$$

and the standard expansion in the $\overline{\mathrm{MS}}$ scheme gives

$$
\delta_{\mathrm{FO}}^{(0)}=0.1991 \pm 0.0061_{-0.0073}^{+0.0042} \pm 0.0119 .
$$

We note that for the standard expansion in the $C$-scheme, where the optimal $C$ for the central scale is close to -1 [10], the errors due to scale variations have been calculated with the second prescription discussed above, and may be underestimated. We mention finally that for the Borelimproved expansions the uncertainty (21) due to the regularization prescription turns out to be 0.0157 for the $C$ scheme and 0.0080 for $\overline{\mathrm{MS}}$.

For the calculation in CIPT, we use Eq. (42) and the numerical procedure described at the end of Sec. V. In Fig. 5 we show the variation with $C$ of the Borel-improved expansion of $\delta_{\mathrm{CI}}^{(0)}$ calculated to order $\alpha_{s}^{5}$ in the $C$ scheme, and also the curves obtained by either removing or 


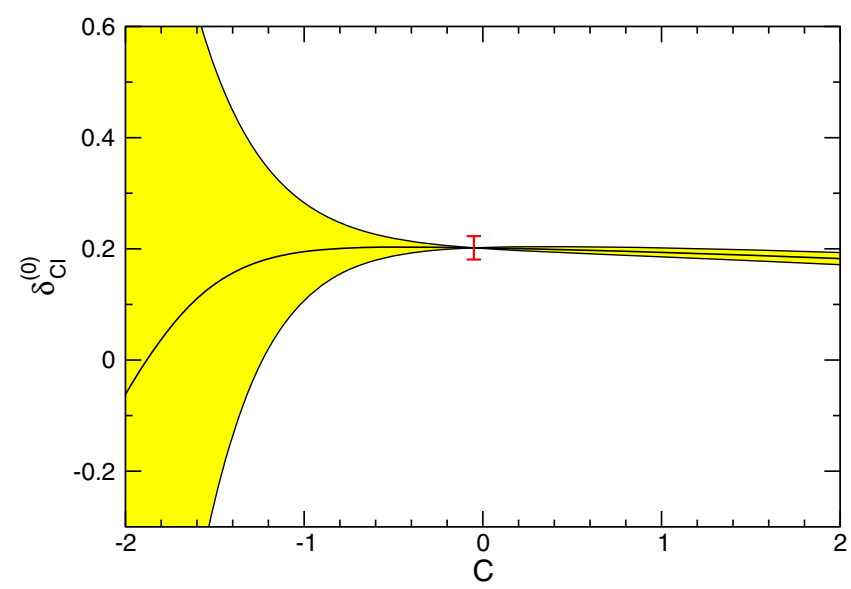

FIG. 5. Borel-improved expansion of $\delta_{\mathrm{CI}}^{(0)}$ to $O\left(\alpha_{s}^{5}\right)$ in the $C$ scheme as a function of $C$. The yellow band arises from either removing or doubling the fifth-order term. In red is marked the point where the last term in the expansion vanishes, and the magnitude of the previous term is indicated.

doubling the last coefficient $\hat{d}_{4}(C)$. For the scale $\mu^{2}=-s$ these curves intersect each other at $C_{0}=-0.05$, where $\hat{d}_{4}(C)$ vanishes. By varying also the scale as $\mu^{2}=-\xi s$, we obtain:

$$
\delta_{\mathrm{CI}}^{(0)}=0.2018 \pm 0.0211_{-0.0139}^{+0.0008} \pm 0.0123
$$

where, as above, the first error is the magnitude of the last nonvanishing term kept in the expansion, the second is due to scale variation and the third accounts for the uncertainty in the coupling. For comparison, the Borel-improved expansion in the $\overline{\mathrm{MS}}$ scheme gives

$$
\delta_{\mathrm{CI}}^{(0)}=0.1997 \pm 0.0018_{-0.0054}^{+0.0007} \pm 0.0119,
$$

while the standard expansion in the $C$-scheme predicts

$$
\delta_{\mathrm{CI}}^{(0)}=0.1840 \pm 0.0062_{-0.0044}^{+0.0002} \pm 0.0084,
$$

and the standard expansion in the $\overline{\mathrm{MS}}$ scheme gives:

$$
\delta_{\mathrm{CI}}^{(0)}=0.1826 \pm 0.0032_{-0.0029}^{+0.0004} \pm 0.0082 .
$$

We note that for the standard expansion in the $C$-scheme, where the optimal $C$ for the central scale is large and negative [10], the errors due to scale variations have been calculated with the second prescription discussed above, and may be underestimated. The prescription ambiguity for the Borel-improved expansions turns out to be very small in this case, below 0.0011 for both $C$ and $\overline{\mathrm{MS}}$ schemes.

From (47) and (51) it is seen that the difference between the FOPT and CIPT predictions for $\delta^{(0)}$ persists also for the Borel-improved expansions in the $C$-scheme. On the other hand, one can notice the close results obtained with the
Borel-improved $\mathrm{CI}$ expansions and the standard FO expansions, for both renormalization schemes: for the $C$-scheme this can be seen by comparing the central values of (49) and (51), and for $\overline{\mathrm{MS}}$ by comparing the central values of (50) and (52). Note that the standard FOPT in the $C$-scheme and the Borel-improved CIPT in the $C$-scheme lead to results very close to the value obtained with the mathematical model [27] presented in the Appendix, which is $\delta^{(0)}=0.2047 \pm 0.0029 \pm 0.0130$.

One can understand these results by a closer examination of the two expansions (a detailed discussion is given in [23]). The good predictions of the standard FOPT are actually due to some fortuitous cancellations between the contributions of large terms in the integral (7) along the circle. By the conformal mapping of the Borel plane, which improves the series convergence, the large coefficients of the FO series near the timelike axis are no longer compensated to the same extent. Therefore, the Borelimproved FO expansion is expected to give poorer results. By contrast, in the Borel-improved CI summation, the improvement of the series convergence is combined with the exact renormalization group summation of the running coupling along the circle $|s|=m_{\tau}^{2}$, ensuring a good convergence of the series (9) along the whole integration contour. Therefore, FOPT appears to be the good choice for the standard expansions, while CIPT is the preferred choice for the Borel-improved expansions in both $\overline{\mathrm{MS}}$ and $C$ schemes. With these options, the results of the FO and CI predictions for the $\tau$ decay width are compatible within errors.

\section{High-order behavior}

The high-order behavior of the standard QCD perturbation expansions in the $C$-scheme has been investigated in $[11,12]$, where a realistic renormalon-based model of the Adler function, proposed in [27], was adopted for generating the higher-order perturbative coefficients. We use the same model (presented for completeness in the Appendix) for assessing the quality of the Borel-improved expansions in the $C$-scheme. We mention that the high-order behavior of the Borel-improved expansions in the $\overline{\mathrm{MS}}$ scheme has been discussed in $[18,21,23]$.

In Fig. 6 we present the variation with $C$ of the Borelimproved expansions in the $C$-scheme of the Adler function $\hat{D}$ at the spacelike point $s=-m_{\tau}^{2}$, for increasing orders of perturbation theory ( $N$ denotes the number of terms kept in the expansion). In this calculation we used the expansion (27) of the Borel transform, based on the optimal conformal mapping (22) and the softening factors expressed in the variable $w$. The scale was fixed at $\mu=m_{\tau}$ and the perturbative coefficients of the model in $\overline{\mathrm{MS}}$ scheme, given in Eq. (A6), have been used as input.

The curves shown in Fig. 6 exhibit a common region of stability with respect to $C$ and a remarkable convergence of 


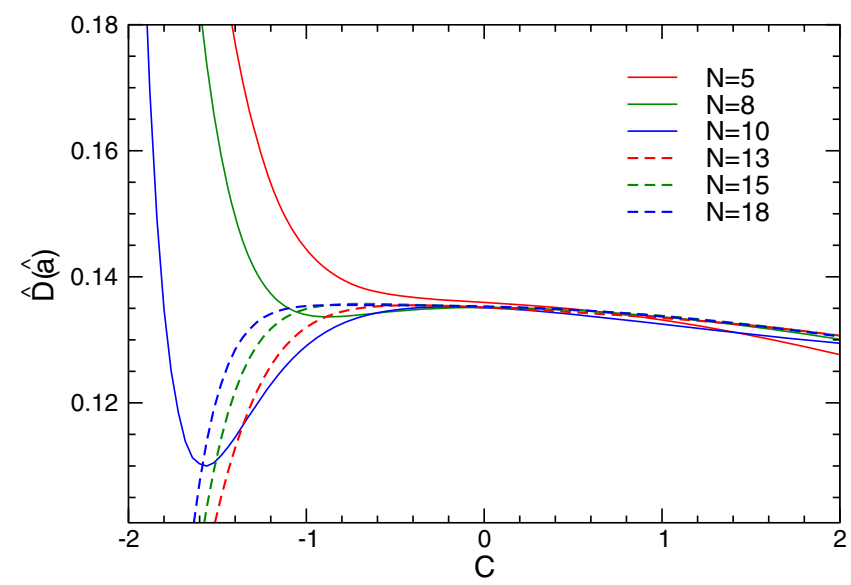

FIG. 6. Variation with $C$ of the Borel-improved expansions in the $C$-scheme of the Adler function $\hat{D}$ at $s=-m_{\tau}^{2}$, for various truncation orders $N$.

the truncated expansions in this region. The bigger variations which appear when $C$ is decreased towards the lower limit of the chosen range are due to the fact that in this range the perturbative connection between the QCD coupling in the $\overline{\mathrm{MS}}$ and $C$ schemes breaks down, so the use of perturbation theory is not legitimate.

For a detailed numerical comparison, we present in Table I the values of the Adler function calculated at $s=$ $-m_{\tau}^{2}$ with the scale $\mu=m_{\tau}$, using several perturbative expansions in the $C$-scheme discussed in Sec. IV. The aim was to assess the relative effect of the renormalon softening and the conformal mapping in improving the convergence. Thus, besides the standard perturbative expansion and the optimal expansion based on both conformal mapping and renormalon softening, we investigated expansions of $B(u)$ in powers of the Borel variable $u$, with the first UV renormalon removed, the first IR renormalon removed and both UV and IR renormalons removed, as well as the expansion in the optimal conformal variable $w$ with no renormalon softening. The equations specifying these expansions are indicated for each column of Table I.

An open problem in the analysis is the choice of the value of $C$ to be used in the expansions. Several values have been considered in Refs. [11,12] for the calculation of the Adler function and the quantity $\delta^{(0)}$ using the standard expansions in the $C$-scheme, which exhibit a divergent behavior.

In the present work we used, for each expansion, the value of $C$ determined in subsection VI A as the optimal $C$ for the corresponding expansion truncated at $N=5$, which means that the last term of the expansion with $N=5$ was set to zero. This explains why in Table I the expansions with $N=4$ and $N=5$ coincide (except for columns 4 and 8 , where no solution of this condition was found, and the value of $C$ minimizing the fifth term was adopted instead).

As shown in Fig. 6, the Borel-improved expansions exhibit a common region of stability for all orders $N$, so the choice of a single $C$ in this region is reasonable at least for these expansions. Of course, an optimal $C$ can be calculated for each truncation order $N$ (provided the condition has acceptable solutions), and an order-dependent $C$ can be used in practical applications. We recall that, while in the standard expansion the condition can be achieved only by the vanishing of the coefficient $\hat{d}_{N}$, in the expansions based on the Borel transform the expansion function $\hat{\mathcal{W}}_{N}$ itself can vanish. In our numerical study, it turned out that the

TABLE I. Adler function $\hat{D}$ of the model [27] calculated at $s=-m_{\tau}^{2}$ with perturbative expansions in the $C$-scheme truncated at order $N$. Second column: standard expansion. Next three columns: expansions of Borel transform in powers of $u$ with first renormalons accounted for. Last four columns: expansions of the Borel transform in the optimal variable $w$, without and with renormalon softening. Exact value of the model: $\hat{D}=0.1354$.

\begin{tabular}{lcccccccc}
\hline \hline$N$ & Eq. (15) & Eq. (30) & Eq. (31) & Eq. (32) & Eq. (23) & Eq. (28) & Eq. (29) & Eq. (27) \\
\hline 3 & 0.1273 & 0.1329 & 0.1247 & 0.1266 & 0.1256 & 0.1401 & 0.1232 & 0.1280 \\
4 & 0.1343 & 0.1379 & 0.1293 & 0.1358 & 0.1441 & 0.1372 & 0.1280 & 0.1360 \\
5 & 0.1343 & 0.1379 & 0.1331 & 0.1358 & 0.1441 & 0.1372 & 0.1324 & 0.1360 \\
6 & 0.1414 & 0.1268 & 0.1359 & 0.1371 & 0.1435 & 0.1337 & 0.1361 & 0.1360 \\
7 & 0.1377 & 0.1560 & 0.1388 & 0.1344 & 0.1439 & 0.1371 & 0.1391 & 0.1357 \\
8 & 0.1567 & 0.0949 & 0.1421 & 0.1359 & 0.1363 & 0.1338 & 0.1412 & 0.1351 \\
9 & 0.1283 & 0.1998 & 0.1462 & 0.1348 & 0.1253 & 0.1352 & 0.1418 & 0.1350 \\
10 & 0.2279 & 0.0652 & 0.1515 & 0.1362 & 0.1181 & 0.1354 & 0.1405 & 0.1352 \\
11 & -0.0195 & 0.1176 & 0.1599 & 0.1355 & 0.1177 & 0.1347 & 0.1378 & 0.1351 \\
12 & 0.8446 & 0.5601 & 0.1724 & 0.1340 & 0.1192 & 0.1355 & 0.1347 & 0.1351 \\
13 & -2.004 & -1.607 & 0.1962 & 0.1413 & 0.1238 & 0.1353 & 0.1326 & 0.1352 \\
14 & 8.982 & 5.581 & 0.2347 & 0.1413 & 0.1300 & 0.1353 & 0.1321 & 0.1353 \\
15 & -34.61 & -14.86 & 0.3288 & 0.1706 & 0.1305 & 0.1355 & 0.1332 & 0.1353 \\
16 & 154.94 & 38.25 & 0.4724 & 0.0594 & 0.1273 & 0.1353 & 0.1351 & 0.1353 \\
17 & -711.57 & -90.63 & 0.9929 & 0.2902 & 0.1247 & 0.1354 & 0.1367 & 0.1353 \\
18 & 3522.7 & 202.12 & 1.4979 & -0.1629 & 0.1229 & 0.1354 & 0.1372 & 0.1353 \\
\hline \hline
\end{tabular}



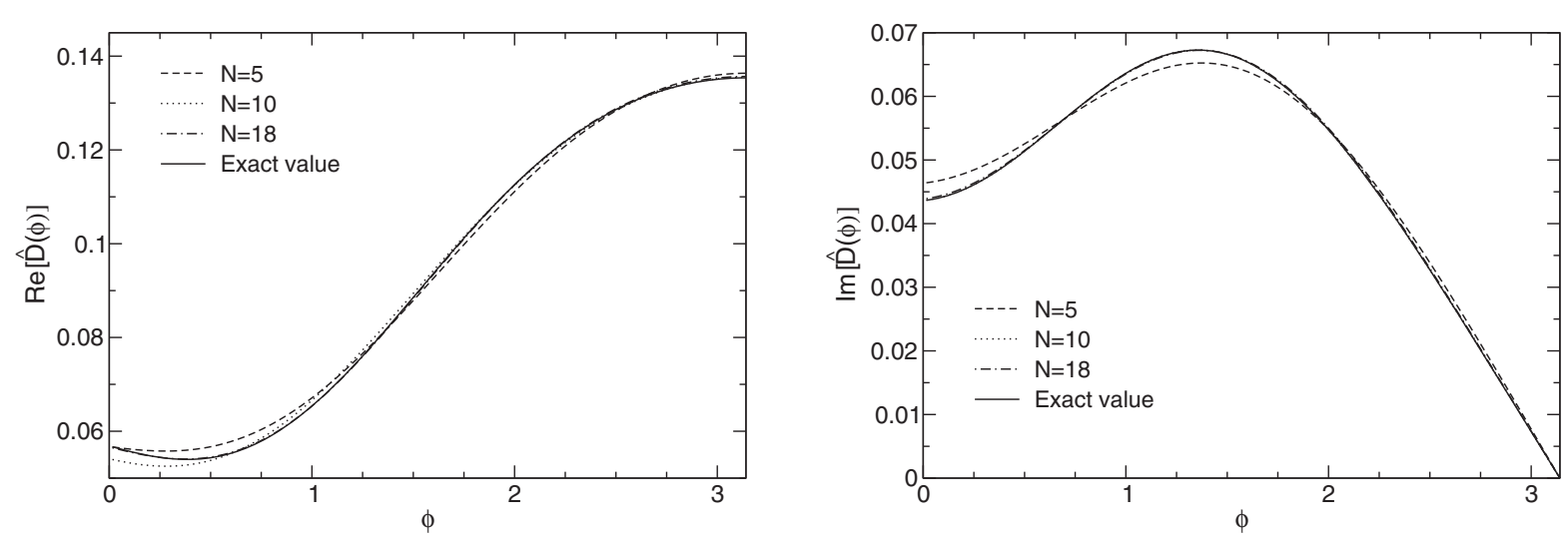

FIG. 7. Real part (left) and imaginary part (right) of the Adler function in the model [27] and its truncated Borel-improved expansions in the $C$-scheme along the circle $|s|=m_{\tau}^{2} \exp (i \phi)$.

condition was achieved in most cases through the vanishing of the expansion function.

From Table I one can remark the divergent behavior of the standard expansion in the $C$-scheme, given in column 2 . Column 3 shows that by softening the first IR renormalon at $u=2$, the low perturbative orders are improved, but the series is badly divergent at large orders. This is expected actually, because the first UV renormalon, which is closest to the origin of the Borel plane, limits to $|u|<1$ the convergence region of the expansion (30). From column 4 it is seen that the effect of softening the first UV renormalon is slightly weaker at low orders, but the divergent character at larger orders is considerably tamed, while by softening both renormalons good results are obtained up to relatively high orders. The expansion is however divergent, although this starts to be visible only at larger orders. Finally, from columns 6-9 it is seen that the effect of the conformal mapping is to ensure convergent expansions at high orders. The additional softening factors improve the description at low orders, the softening of the first IR renormalon having a more visible effect than that of the first UV renormalon. We note that by using order-dependent optimal values of $C$, determined from the optimum condition at each order $N$, the convergence of the expansions based on the conformal mapping towards the exact result of the model is even more precise than shown in columns 6-9 of Table I.

The large-order behavior of the expansions of the $\tau$ hadronic width has been also investigated, in the $\overline{\mathrm{MS}}$ scheme in $[21,23,27]$, and in the $C$-scheme in $[11,12]$. As discussed in Sec. II, the quantity $\delta^{(0)}$, which involves the values of the Adler function in the complex $s$ plane, allows one to extract only indirect information about the perturbation expansion of the Adler function itself along the circle.

In order to assess in a straightforward way the quality of the expansions in the complex plane, we compare in Fig. 7 the values of the Adler function calculated with the model presented in the Appendix for complex values $s=m_{\tau}^{2} \exp (i \phi)$, and its Borel-improved approximants in the $C$-scheme given by (38), with the running coupling $\hat{a}_{\mu}$ calculated for $\mu^{2}=-s$ by integrating the renormalizationgroup equation in the $C$-scheme, as explained at the end of Sec. V. We restricted $\phi$ to the range $\phi \in(0, \pi)$, the values on the semicircle in the lower half-plane being obtained by using the Schwarz reflection property $\hat{D}\left(s^{*}\right)=\hat{D}^{*}(s)$. The calculations have been done with the value $C=-0.05$, which is the optimal choice for the evaluation of the Adler function at $s=-m_{\tau}^{2}$ with $N=5$ terms in the expansion. One can remark the impressive convergence along the whole circle $|s|=m_{\tau}^{2}$ of the Borel-improved expansions in the $C$-scheme, up to high perturbative orders. By inserting these expansions into the definition (7) of $\delta^{(0)}$, one obtains good predictions for this quantitity in CIPT, which, as discussed in the previous subsection, is the preferred choice of summation for the Borel-improved expansions. As shown in [21,23], a similar behavior is obtained for the Borel-improved expansions in $\overline{\mathrm{MS}}$ scheme, while the standard expansions show big oscillations far from the true values.

\section{DISCUSSION AND CONCLUSIONS}

In the present paper we investigated the renormalization scheme variation of an improved perturbation expansion in QCD, with tamed behavior at large orders, defined by means of the optimal conformal mapping of the Borel plane. Detailed studies performed in previous works [21-25] demonstrated the good properties of these Borel-improved expansions in the $\overline{\mathrm{MS}}$ scheme. The analysis was extended now to a new renormalization scheme, denoted as $C$-scheme, proposed in [10] and investigated in the frame of standard QCD expansions in [10-12]. Our purpose was not to advocate the advantage of a particular scheme, but to study the variation with the renormalization scheme of a quantity known to be renormalization-scheme invariant. We performed our study using as examples the perturbation expansions of the Adler function and of the $\tau$ hadronic width. 
For the expansions to order $\alpha_{s}^{5}$ and higher, we found a range of stability of the results with respect to the variation of the $C$ parameter defining the RS, shown in Figs. 2-6. In Ref. [10], the optimal value of $C$ was defined by requiring that the highest term in the expansion vanishes. Moreover, in Ref. [10], an additional assumption was made, namely the last nonzero term in the expansion was taken as the truncation error to be attached to the central prediction. We adopted in the present work the same prescriptions, but found that they might be too rigid in some situations. Therefore, the values quoted as uncertainties in Sec. VI for the predictions of the Adler function and the phenomenological parameter $\delta^{(0)}$ in the $C$-scheme must be taken with some caution.

One problem is the danger of a distorted truncation uncertainty, due to accidentally large or small values of the last nonzero term. Another problem was revealed when studying the dependence of the results on the renormalization scale $\mu$ : namely, if the optimal $C$ for the central value of $\mu$ is negative and large, it may not be suitable for lower values of $\mu$, for which the coupling is too large (as seen from Fig. 1), and the perturbation expansion breaks down. We note also that the optimum prescription proposed in [10] cannot be applied to expansions at lower orders, $N=3$ and $N=4$. For the standard expansions in the $C$-scheme, no solution of the condition exists, as the three curves corresponding to Fig. 2 do not intersect each other. For the Borel-improved expansion in the $C$-scheme, a common intersection point exists, but it is situated in a region of large negative $C$, where the variation with respect to $C$ is not stable.

These problems suggest that a more elaborate definition of the optimal $C$ and of the theoretical error might be necessary. It would be reasonable, for instance, to require the simultaneous fulfillment of several conditions: smallness of the last term in the expansion (instead of requiring an exact zero), stability with respect to the variation of $C$, and stability with respect to the variation of the scale. Such a study, necessary for phenomenological applications, in particular for a precise determination of $\alpha_{s}$ from hadronic $\tau$ decays, is beyond the scope of the present paper, whose main aim was to investigate the behavior of the Borelimproved expansions in the $C$-scheme.

For the parameter $\delta^{(0)}$, we found in the $C$-scheme a property already noticed in $\overline{\mathrm{MS}}$ scheme, namely the close results given by the CIPT Borel-improved expansions and the FOPT standard expansions. Therefore, FOPT appears to be the good choice for the standard expansions, while CIPT is the preferred choice for the Borel-improved expansions in both $\overline{\mathrm{MS}}$ and $C$ schemes.

The good properties of the Borel-improved expansions manifest themselves in an impressive way at large orders. In our study, for generating the higher perturbative coefficients we used as in [10-12] a theoretical model for the Adler function, proposed in [27]. The results shown in Figs. 6 and 7 prove the remarkable convergence of the Borel- improved expansions in the $C$-scheme of the Adler function evaluated on the Euclidian axis and in the complex $s$ plane.

In order to assess the relative effects of the singularitysoftening factors and of the conformal mapping of the Borel plane, we investigated also several other expansions, in which only the singularity softening is incorporated, with no conformal mapping in the power expansion, or only the conformal mapping without singularity softening is used. The results given in Table I show that the proper treatment of lowest renormalons improves the low-orders but cannot cure the divergence at high orders, while the use of the optimal conformal mapping without softening factors ensures the convergence at high orders, but may give poorer results at low orders. Finally, the Borel-improved expansion based on singularity-softening factors and the optimal conformal mapping of the Borel plane gives good results at low orders and converges towards the true values at large orders.

As discussed in Sec. VI, an open problem for the expansions in the $C$-scheme is the proper choice of the parameter $C$ which defines a particular RS. We argued that an order-dependent optimal $C$ appears to be the best choice, and emphasized that in the Borel-improved expansion the optimum condition can be achieved also by imposing the vanishing of the last expansion function, a possibility that does not exist for the standard expansion.

We emphasize finally that the model used in the present study for generating higher-order perturbative coefficients was constructed in [27] from a renormalon analysis in $\overline{\mathrm{MS}}$ scheme. Moreover, the free parameters of the model are determined such as to generate the lowest perturbative coefficients (5) known in $\overline{\mathrm{MS}}$ scheme. However, we proved that the Borel-improved expansions converge to the exact result even if the expansions are defined in the $C$-scheme. This provides a nice illustration of the renormalizationscheme independence of the QCD perturbation theory, once the large-order divergence is properly treated.

The results of the present analysis are a further argument in favor of the nonpower expansions based on the optimal conformal mapping of the Borel plane, which prove to be a useful tool for applications of perturbative QCD at intermediate energies.

\section{ACKNOWLEDGMENTS}

I thank D. Boito for useful discussions and suggestions on the manuscript. This work was supported by the Romanian Ministry of Research and Innovation, Contract No. PN 18090101/2018.

\section{APPENDIX: MODEL OF THE ADLER FUNCTION}

For testing the convergence of the various expansions, we considered the model proposed in [27], which expresses the Adler function by means of the PV-regulated LaplaceBorel integral: 


$$
\hat{D}\left(a_{\mu}\right)=\frac{1}{\beta_{0}} \mathrm{PV} \int_{0}^{\infty} e^{-\frac{u}{\beta_{0} a_{\mu}}} B(u) \mathrm{d} u
$$

with a Borel transform $B(u)$ parametrized in terms of a few UV and IR renormalons. Specifically, in the model proposed in [27], the function $B(u)$ is expressed as

$B(u)=B_{1}^{\mathrm{UV}}(u)+B_{2}^{\mathrm{IR}}(u)+B_{3}^{\mathrm{IR}}(u)+d_{0}^{\mathrm{PO}}+d_{1}^{\mathrm{PO}} u$,

where

$$
\begin{aligned}
B_{p}^{\mathrm{IR}}(u) & =\frac{d_{p}^{\mathrm{IR}}}{(p-u)^{\gamma_{p}}}\left[1+\tilde{b}_{1}(p-u)+\ldots\right], \\
B_{p}^{\mathrm{UV}}(u) & =\frac{d_{p}^{\mathrm{UV}}}{(p+u)^{\bar{\gamma}_{p}}}\left[1+\bar{b}_{1}(p+u)+\ldots\right] .
\end{aligned}
$$

The free parameters of the models are the residues $d_{1}^{\mathrm{UV}}$, $d_{2}^{\mathrm{IR}}$ and $d_{3}^{\mathrm{IR}}$ of the first renormalons and the coefficients $d_{0}^{\mathrm{PO}}, d_{1}^{\mathrm{PO}}$ of the polynomial in (A2), determined in [27] as

$d_{1}^{\mathrm{UV}}=-1.56 \times 10^{-2}, \quad d_{2}^{\mathrm{IR}}=3.16, \quad d_{3}^{\mathrm{IR}}=-13.5$,

$d_{0}^{\mathrm{PO}}=0.781, \quad d_{1}^{\mathrm{PO}}=7.66 \times 10^{-3}$, by the requirement to reproduce the perturbative coefficients $c_{n, 1}$ in $\overline{\mathrm{MS}}$ scheme for $n \leq 4$, given in (5), and the estimate $c_{5,1}=283$.

Once the parameters are fixed, the model predicts all the higher order perturbative coefficients $c_{n, 1}$ for $n>5$. We give below the values of the coefficients used in the calculations presented in Sec. VI: singularity-softening factors and of the conformal mapping of the Borel plane

$$
\begin{array}{rlrl}
c_{6,1} & =3275.45, \quad c_{7,1}=18758.4, \quad c_{8,1}=388446, \\
c_{9,1} & =919119, \quad c_{10,1}=8.36 \times 10^{7}, \\
c_{11,1} & =-5.19 \times 10^{8}, & c_{12,1}=3.38 \times 10^{10}, \\
c_{13,1} & =-6.04 \times 10^{11}, & c_{14,1}=2.34 \times 10^{13}, \\
c_{15,1} & =-6.52 \times 10^{14}, & c_{16,1} & =2.42 \times 10^{16}, \\
c_{17,1} & =-8.46 \times 10^{17}, & c_{18,1} & =3.36 \times 10^{19} .
\end{array}
$$

One can note the dramatic increase of the coefficients, which implies that the perturbation series of the Adler function in this model is divergent.

For $\alpha_{s}\left(m_{\tau}^{2}\right)=0.316 \pm 0.010$, the Adler function at $s=$ $-m_{\tau}^{2}$ and $\mu=m_{\tau}$ given by this model has the value [10]: $\hat{D}\left(a_{m_{\tau}}\right)=0.1354 \pm 0.0127 \pm 0.0058$, where the first error comes from renormalon ambiguity, evaluated using the prescription (21) and the second from the uncertainty of the coupling.
[1] P. M. Stevenson, Phys. Rev. D 23, 2916 (1981).

[2] G. Grunberg, Phys. Rev. D 29, 2315 (1984).

[3] S. Brodsky, P. Lepage, and P. Mackenzie, Phys. Rev. D 28, 228 (1983).

[4] J. Chyla, A. Kataev, and S. Larin, Phys. Lett. 267, 269 (1991).

[5] S. J. Brodsky and L. Di Giustino, Phys. Rev. D 86, 085026 (2012).

[6] S. J. Brodsky and X. G. Wu, Phys. Rev. Lett. 109, 042002 (2012).

[7] M. Mojaza, S. J. Brodsky, and X. G. Wu, Phys. Rev. Lett. 110, 192001 (2013).

[8] J. M. Shen, X. G. Wu, B. L. Du, and S. J. Brodsky, Phys. Rev. D 95, 094006 (2017).

[9] W. A. Bardeen, A. J. Buras, D. W. Duke, and T. Muta, Phys. Rev. D 18, 3998 (1978).

[10] D. Boito, M. Jamin, and R. Miravitllas, Phys. Rev. Lett. 117, 152001 (2016).

[11] D. Boito, M. Jamin, and R. Miravitllas, EPJ Web Conf. 137, 05007 (2017).

[12] D. Boito, M. Jamin, and R. Miravitllas, Nucl. Part. Phys. Proc. 287, 77 (2017).
[13] X.-G. Wu, J.-M. Shen, B.-L. Du, and S. J. Brodsky, Phys. Rev. D 97, 094030 (2018).

[14] G. 't Hooft, in The Whys of Subnuclear Physics, Proceedings of the 15th International School on Subnuclear Physics, Erice, Sicily, 1977, edited by A. Zichichi (Plenum Press, New York, 1979), p. 943.

[15] A. H. Mueller, Nucl. Phys. B250, 327 (1985).

[16] A. H. Mueller, in QCD-Twenty Years Later, Aachen 1992, edited by P. Zerwas and H. A. Kastrup (World Scientific, Singapore, 1992).

[17] M. Beneke, Phys. Rep. 317, 1 (1999).

[18] I. Caprini and J. Fischer, Phys. Rev. D 60, 054014 (1999).

[19] I. Caprini and J. Fischer, Phys. Rev. D 62, 054007 (2000).

[20] I. Caprini and J. Fischer, Eur. Phys. J. C 24, 127 (2002).

[21] I. Caprini and J. Fischer, Eur. Phys. J. C 64, 35 (2009).

[22] I. Caprini and J. Fischer, Nucl. Phys. B, Proc. Suppl., 218, 128 (2011).

[23] I. Caprini and J. Fischer, Phys. Rev. D 84, 054019 (2011).

[24] G. Abbas, B. Ananthanarayan, I. Caprini, and J. Fischer, Phys. Rev. D 87, 014008 (2013).

[25] G. Abbas, B. Ananthanarayan, I. Caprini, and J. Fischer, Phys. Rev. D 88, 034026 (2013). 
[26] I. Caprini, J. Fischer, G. Abbas, and B. Ananthanarayan, in Perturbation Theory: Advances in Research and Applications (Nova Science Publishers, Hauppauge, New York, 2018), p. 211.

[27] M. Beneke and M. Jamin, J. High Energy Phys. 09 (2008) 044.

[28] P. A. Baikov, K. G. Chetyrkin, and J. H. Kühn, Phys. Rev. Lett. 118, 082002 (2017).

[29] P. A. Baikov, K. G. Chetyrkin, and J. H. Kühn, Phys. Rev. Lett. 101, 012002 (2008).

[30] S. Narison and A. Pich, Phys. Lett. B 211, 183 (1988).

[31] E. Braaten, Phys. Rev. Lett. 60, 1606 (1988).

[32] E. Braaten, S. Narison, and A. Pich, Nucl. Phys. B373, 581 (1992).

[33] F. L. Diberder and A. Pich, Phys. Lett. B 286, 147 (1992).

[34] M. R. Ahmady, F. A. Chishtie, V. Elias, A. H. Fariborz, D. G. C. McKeon, T. N. Sherry, A. Squires, and T. G. Steele, Phys. Rev. D 67, 034017 (2003).

[35] G. Abbas, B. Ananthanarayan, and I. Caprini, Phys. Rev. D 85, 094018 (2012).

[36] M. Davier, S. Descotes-Genon, A. Hocker, B. Malaescu, and Z. Zhang, Eur. Phys. J. C 56, 305 (2008).

[37] S. Menke, arXiv:0904.1796.

[38] A. Pich, Acta Phys. Pol. B Proc. Suppl. 3, 165 (2010).

[39] S. Descotes-Genon and B. Malaescu, arXiv:1002.2968.

[40] A. Pich, Nucl. Phys. B, Proc. Suppl. 218, 89 (2011).

[41] A. Pich, arXiv:1110.0016.

[42] A. Pich, Prog. Part. Nucl. Phys. 75, 41 (2014).
[43] M. Beneke, D. Boito, and M. Jamin, J. High Energy Phys. 01 (2013) 125.

[44] A. Pich and A. Rodriguez-Sánchez, Phys. Rev. D 94, 034027 (2016).

[45] D. Boito, M. Golterman, K. Maltman, and S. Peris, Phys. Rev. D 95, 034024 (2017).

[46] I. Caprini and M. Neubert, J. High Energy Phys. 03 (1999) 007.

[47] S. Ciulli and J. Fischer, Nucl. Phys. 24, 465 (1961).

[48] W. R. Frazer, Phys. Rev. 123, 2180 (1961).

[49] I. Ciulli, S. Ciulli, and J. Fischer, Nuovo Cim. 23, 1129 (1962).

[50] R. Seznec and J. Zinn-Justin, J. Math. Phys. 20, 1398 (1979).

[51] J. Zinn-Justin and U. D. Jentschura, J. Math. Phys. 51, 072106 (2010).

[52] G. Altarelli, P. Nason, and G. Ridolfi, Z. Phys. C 68, 257 (1995).

[53] D. E. Soper and L. R. Surguladze, Phys. Rev. D 54, 4566 (1996).

[54] M. Beneke, V. M. Braun, and N. Kivel, Phys. Lett. B 404, 315 (1997).

[55] A. Pineda, J. High Energy Phys. 06 (2001) 022.

[56] A. H. Hoang, A. Jain, I. Scimemi, and I. W. Stewart, Phys. Rev. D 82, 011501 (2010).

[57] G. Cvetič and T. Lee, Phys. Rev. D 64, 014030 (2001).

[58] M. Tanabashi et al. (Particle Data Group), Phys. Rev. D 98 , 030001 (2018). 